\title{
A Robust Adaptive Beamformer Based on Semidefinite Programming with Quadratic Constraints
}

\author{
Feng Shen and Jinyang Song \\ College of Automation, Harbin Engineering University, Harbin, China \\ sf407@126.com
}

\begin{abstract}
A robust beamforming with quadratic constraints, formulated as a semidefinite programming (SDP) problem, is proposed in this paper. With this formulation, the constraints on magnitude response can be easily imposed on the adaptive beamformer. And the non-convex quadratic constraints can be transformed into linear constraints. Therefore, the proposed method can be robust against the steering direction error. In practice, there are many array imperfections except steering direction error. In order to resist all kinds of array imperfections, the adaptive beamformer based on worst-case optimization technique is proposed by minimizing the array output power with respect to the worst-case array imperfections. Simulation results demonstrate that the proposed method is effective and can achieve a better performance.
\end{abstract}

Keywords: Adaptive array; Robust adaptive beamforming; Semidefinite programming; Worst-case optimization

\section{Introduction}

Array signal processing is widely used in many fields such as radar, communication, sonar, acoustics, medical imaging and so on. Beamforming is an important assignment in array processing applications. A well-known representative, named the Capon beamformer [1] constrains the response of the signal of interest (SOI) to be unity and minimizes the variance of the beamformer output. The Capon beamformer has better resolution and interference rejection capability if the array steering vector corresponding to the SOI is known accurately.

In practical applications, the array steering vector (ASV) of the SOI can be imprecise due to the existence of array imperfections, such as steer direction errors, local scattering, near-far spatial signature mismatch, waveform distortion, source spreading, imperfectly calibrated arrays and distorted antenna shape [2]. Adaptive array is very sensitive to the ASV errors, which cause serious target signal cancellation problem and result in array performance drastic degradation, especially for the array output signal-to-interference-plus-noise ratio (SINR) [3]. In such cases, robust approaches to adaptive beamforming are required.

Many methods have been proposed to make the adaptive beamformer robust against ASV errors. In [4-8], linear constraints have been imposed when minimizing the output variance, which can be designed to broaden the main beam of adaptive array. These beamformers are the so-called linearly constrained minimum variance (LCMV) beamformers. Convex quadratic constraints [9-10], a Bayesian approach [11], also have been proposed to make the array robust against steer direction error. However, the applicability of these techniques is limited by scenarios with the steering direction error of the SOI only. Considering more general array imperfections, diagonal loading method [3] is known to provide robustness. But the main problem of the conventional diagonal loading method is how to determine the level of diagonal loading. Another popular approach to robust adaptive beamforming is the 
eigenspace-based beamformer [12-13]. Nevertheless, it is ineffective at low signal-to-noise ratio (SNR) and when the dimension of the signal-plus-interference subspace is high or not exactly known, the performance of eigenspace-based beamformer degrades drastically.

Recently, there are some robust methods with clear theoretical background have been proposed [14-17]. The designed beamformers are robust against general types of ASV errors with an assumed uncertainty set. The uncertainty set has been selected as polyhedron, sphere, or ellipsoid, and these beamformers are equivalent to and belong to the diagonal loading method [16]. The diagonal loading level can be calculated based on the known level of uncertainty of the signal steering vector. In [18], inspired by these uncertainty-based methods, a simplified uncertainty set has been considered, which contains only the steering vectors with a desired uncertainty range of direction-of-arrival. However, it is not clear how to generate the array weight because the constraint is an infinite number of non-convex quadratic constraints. In [18], a suboptimal solution has been derived by loosening the constraint.

In this paper, we consider an alternative and efficient approach to solve the robust beamformer with non-convex quadratic constraints. We formulate the robust adaptive beamformer in the form of an SDP problem. With this formulation, the constraints on magnitude response can be easily imposed on the adaptive beamformer. The non-convex quadratic constraints can be transformed into linear constraints. With the quadratic constraints, the method is only robust against the steering direction error. In order to ensure the robustness against arbitrary array imperfections, the technique of worst-case optimization is used to enhance the robustness against arbitrary array imperfections and outstanding SINR improvement. The approach is to find the worst effect of the array imperfection on the array output power, and minimizes the array output power according to the worst-case array imperfection. By ignoring the rank-one constraint, the proposed SDP with quadratic constraints based on worst-case optimization can be formulated as a relaxed SDP problem, which can be solved efficiently by using the interior-point method [19] with some open solvers, e.g. CVX [20] and SeDuMi [21] available.

The notations adopted hereafter are standard. $(\cdot)^{T}$ denotes the transpose operator, $(\cdot)^{H}$ denotes the Hermitian transpose operator, $E\{\cdot\}$ denotes the mathematical expectation, and $\operatorname{tr}\{\cdot\}$ denotes the trace operator.

\section{Robust Adaptive Beamfromer based on Semidefinite Programming}

\subsection{Background}

Consider a uniform linear array (ULA) of $N$ isotropic sensors with inter-element spacing $d$. The SOI is a narrowband plane wave with wavelength $\lambda$ impinging form angle $\theta$. The received sensor-sampled complex signals (array snapshot) $x(n)=\left[x_{0}(n), \ldots, x_{N-1}(n)\right]^{T}$ is linearly combined with complex weight $w=\left[w_{0}, \ldots, w_{N-1}\right]^{T}$ to yield the beamformer output

$$
y(n)=\boldsymbol{w}^{H} \boldsymbol{x}(n)
$$

Conventional adaptive beamforming method, e.g., the Capon beamformer minimizes array output power while constraining the SOI response to be unity. If the direction $\theta$ is accurately known, the Capon beamformer is formulated as the following optimization problem

$$
\begin{array}{ll}
\min _{\boldsymbol{w}} & \boldsymbol{w}^{H} \boldsymbol{R} \boldsymbol{w} \\
\text { s.t. } & \mathbf{s}^{H}(\theta) \boldsymbol{w}=1
\end{array}
$$


where $\boldsymbol{R}=E\left\{\boldsymbol{x}(n) \boldsymbol{x}(n)^{H}\right\}$ is the covariance matrix of array snapshot. $\boldsymbol{s}(\theta)$ is the ASV of the SOI, and

$$
\boldsymbol{s}(\theta)=\left[\begin{array}{llll}
1 & \mathrm{e}^{j(2 \pi / \lambda) d \sin \theta} & \ldots & \mathrm{e}^{j(N-1)(2 \pi / \lambda) d \sin \theta}
\end{array}\right]^{T}
$$

The Capon beamformer is very sensitive to ASV mismatches caused by array imperfections. The most typical array imperfection is the steer direction error. If $\theta$ deviates from the true one, the minimization in (2) suppresses the magnitude response of the SOI, the phenomenon is called target signal cancellation. To avoid this, [18] use the constraints on magnitude response force the magnitude responses at a range of arrival angles to exceed unity while minimizing array output power. The optimal robust beamformer problem can be expressed as

$$
\begin{array}{ll}
\min _{w} & \boldsymbol{w}^{H} \boldsymbol{R} \boldsymbol{w} \\
\text { s.t. } & \left|\boldsymbol{s}^{H}(\theta) \boldsymbol{w}\right|^{2} \geq 1, \quad \theta \in\left[\theta_{l}, \theta_{u}\right]
\end{array}
$$

where $\theta_{l}$ and $\theta_{u}$ are the lower and upper bounds of the uncertainty of SOI arrival angle with a high probability. It is not clear how to solve the optimal beamformer $w$ in (4) because the magnitude constraints are an infinite number of nonconvex quadratic constraints over $w$, which implies that an optimization problem with such constraints cannot be solved by the well developed convex optimization technique.

\subsection{Robust Adaptive Beamfromer based on Semidefinite Programming with Magnitude Response Constraints}

In this paper, we will formulate the robust adaptive beamformer in the form of a SDP problem. With this formulation, the constraints on magnitude response can be easily imposed on the adaptive beamformer. The non-convex quadratic constraints can be transformed into linear constraints. The array output power in (2) can be expressed as

$$
\boldsymbol{w}^{H} \boldsymbol{R} \boldsymbol{w}=\operatorname{tr}\left\{\boldsymbol{w}^{H} \boldsymbol{R} \boldsymbol{w}\right\}=\operatorname{tr}\{\boldsymbol{R} \boldsymbol{W}\}
$$

where the matrix $\boldsymbol{W}$ is defined as $\boldsymbol{W}=\boldsymbol{w} \boldsymbol{w}^{H}$. It is clear that the rank of $\boldsymbol{W}$ should be constrained to one, i.e., $\operatorname{rank}(\boldsymbol{W})=1 . \boldsymbol{W}$ is the Hermitian positive semidefinite matrix and denoted as

$$
\boldsymbol{W} \succeq 0
$$

Since the magnitude response can be transformed as

$$
\left|\boldsymbol{s}^{H}(\theta) \boldsymbol{w}\right|^{2}=\operatorname{tr}\left\{\boldsymbol{s}(\theta) \boldsymbol{s}^{H}(\theta) \boldsymbol{w} \boldsymbol{w}^{H}\right\}=\operatorname{tr}\left\{\boldsymbol{R}_{s}(\theta) \boldsymbol{W}\right\}
$$

where $\boldsymbol{R}_{s}(\theta)=\boldsymbol{s}(\theta) \boldsymbol{s}^{H}(\theta)$. The constraints in (2) are equivalent to

$$
\operatorname{tr}\left\{\boldsymbol{R}_{s}(\theta) \boldsymbol{W}\right\} \geq 1, \quad \theta \in\left[\theta_{l}, \theta_{u}\right]
$$

From (8), it is clear that this new constraint is linear in $\boldsymbol{W}$. However, the constraint (8) is a semi-infinite constraint. In this paper, it is approximated in a straightforward way by sampling or discretizing the angle. We choose a set of angles such that

$$
\theta_{l} \leq \hat{\theta}_{1} \leq \cdots \leq \hat{\theta}_{k} \leq \theta_{u}
$$

which are often uniformly or logarithmically spaced. The semi-infinite constraint is replaced by a set of ordinary constraints

$$
\operatorname{tr}\left\{\boldsymbol{R}_{s}(\hat{\theta}) \boldsymbol{W}\right\} \geq 1, \quad \hat{\theta} \in \Theta
$$


where $\Theta=\left\{\hat{\theta}_{i} \mid \theta_{l} \leq \hat{\theta}_{i} \leq \theta_{u}, i=1, \ldots, k\right\}$ is a sampled angle set. Combining (5), (10) and $\operatorname{rank}(\boldsymbol{W})=1$, the robust adaptive beamfromer can be formulated as

$$
\begin{aligned}
\min _{w} & \operatorname{tr}\{\boldsymbol{R} \boldsymbol{W}\} \\
& \text { s.t. } \operatorname{tr}\left\{\boldsymbol{R}_{s}(\hat{\theta}) \boldsymbol{W}\right\} \geq 1, \quad \hat{\theta} \in \Theta \\
& \boldsymbol{W} \succeq 0, \operatorname{rank}(\boldsymbol{W})=1
\end{aligned}
$$

which is an equivalent formulation of the beamformer in (4). However, (11) has a non-convex rank-one constraint $\operatorname{rank}(\boldsymbol{W})=1$. It is difficult to solve the problem with non-convex constraint. Using the semidefinite relaxation (SDR) approach [22], we drop the rank-one constraint and relax (11) to the following convex optimization problem

$$
\min _{w} \operatorname{tr}\{\boldsymbol{R} \boldsymbol{W}\} \quad \text { s.t. } \operatorname{tr}\left\{\boldsymbol{R}_{s}(\hat{\theta}) \boldsymbol{W}\right\} \geq 1, \quad \hat{\theta} \in \Theta
$$

which can be solved efficiently by using the interior-point method. Due to the relaxation, the rank of the matrix $\boldsymbol{W}$ obtained by solving the SDR problem may be higher than one. If it is, then the principal eigenvector of $W$ is the optimal weight vector. Otherwise, the optimal weight vector can be recovered from $\boldsymbol{W}$ by using the spectral factorization method [23]. Fortunately, the rank of the matrix $\boldsymbol{W}$ is always one in numerical examples and such spectral factorization procedure does not need.

\section{Robust Adaptive Beamformer based on Worst-case Optimization}

The method described in (12) works well for array with only steering direction errors. However, in practice, due to the limited number of array snapshots and many other array imperfections, the estimated covariance matrix may deviate from the true covariance matrix. When these happen, there is serious performance degradation for the designed adaptive beamformer. In order to ensure the robustness against arbitrary array imperfections, the technique of worst-case optimization is used to enhance the robustness against arbitrary array imperfections and providing outstanding SINR improvement.

We define an error matrix $\Delta$, which takes into account all mismatches that may be caused. With such a general error model, the estimated array covariance matrix $\hat{\boldsymbol{R}}$ can be expressed as

$$
\hat{\boldsymbol{R}}=\boldsymbol{R}-\Delta
$$

where $\boldsymbol{R}$ is the true covariance matrix. The only constraint on the error matrix $\Delta$ is the limited Frobenius norm, i.e., $\|\Delta\| \leq \varepsilon$, where $\varepsilon$ is a positive real number. Similar to [14, 24], we use the idea of the worst-case optimization. The array output power is minimized when the error matrix $\Delta$ brings the worst-case effect on the objective function. Substituting (13) into (4) and using worst-case optimization, the optimization problem is expressed as

$$
\begin{aligned}
& \quad \min _{\boldsymbol{w}} \max _{\Delta} \boldsymbol{w}^{H}(\hat{\boldsymbol{R}}+\Delta) \boldsymbol{w} \\
& \text { s.t. }\left|\boldsymbol{s}^{H}(\theta) \boldsymbol{w}\right|^{2} \geq 1, \quad \theta \in\left[\theta_{1}, \theta_{2}\right]
\end{aligned}
$$

$$
\|\Delta\| \leq \varepsilon
$$


With this new optimization formulation, the robustness of the beamformer should be improved.

To simplify the (14), consider the solution for the following problem

$$
\max _{\Delta} \boldsymbol{w}^{H}(\hat{\boldsymbol{R}}+\Delta) \boldsymbol{w} \text { s.t. }\|\Delta\| \leq \varepsilon
$$

(15)can be rewritten the as

$$
\min _{\Delta}-\boldsymbol{w}^{H}(\hat{\boldsymbol{R}}+\Delta) \boldsymbol{w} \text { s.t. }\|\Delta\| \leq \varepsilon
$$

and using a derivation similar to that given in [24], the solution of (16) is

$$
\Delta=\varepsilon \frac{\boldsymbol{w} \boldsymbol{w}^{H}}{\|\boldsymbol{w}\|^{2}}
$$

and the maximum of the objective function in (14) is

$$
\boldsymbol{w}^{H}(\hat{\boldsymbol{R}}+\varepsilon \boldsymbol{I}) \boldsymbol{w}
$$

Then, using (18), the (14) can be rewritten in a much simpler equivalent form

$$
\min _{w} \boldsymbol{w}^{H}(\hat{\boldsymbol{R}}+\varepsilon \boldsymbol{I}) \boldsymbol{w}
$$

$$
\text { s.t. }\left|\boldsymbol{s}^{H}(\theta) \boldsymbol{w}\right|^{2} \geq 1, \quad \theta \in\left[\theta_{1}, \theta_{2}\right]
$$

Similar to the problem (12), the robust adaptive beamfromer based on semidefinite programming and worst-case optimization can be given as

$$
\begin{aligned}
& \min _{w} \operatorname{tr}\{(\boldsymbol{R}+\varepsilon \boldsymbol{I}) \boldsymbol{W}\} \\
\text { s.t. } & \operatorname{tr}\left\{\boldsymbol{R}_{s}(\hat{\theta}) \boldsymbol{W}\right\} \geq 1, \quad \hat{\theta} \in \Theta
\end{aligned}
$$

$$
\boldsymbol{W} \succeq 0
$$

Its optimal solution is easy to be obtained with the interior-point method (IPM) [19] as same as the method in (12).

\section{Numerical Results}

Analyzing the theoretical performance of general adaptive beamformers is difficult, except some special cases when adaptive beamformers have closed-form solution. Our method also has no closed-form solution. Like other state-of-the-art adaptive beamformers [14-18], we use numerical experiments to evaluate the performance according to the improvement of output SINR. We compare the performance of the proposed method with the performance of the standard Capon beamformer (SCB) [1], the robust Capon beamformer (RCB) [16], Shahbazpanahi's method [24] and Chen's method [18].

We assume a uniform linear array (ULA) with 10 sensors and half-wavelength interelement spacing is used. In all the experiments, the non-directional noise is assumed to be a spatially white Gaussian noise and its covariance matrix is the identity matrix $I$. The power and directions-of-arrival (DOA) of the SOI are $10 \mathrm{~dB}$ and $0^{\circ}$. Two interferences have the power of $20 \mathrm{~dB}$ and DOAs $30^{\circ}$ and $-45^{\circ}$, respectively. All the results are calculated by the average of 200 Monte Carlo trials.

Firstly, we examine the robustness of the beamfomers only in the presence of DOA mismatch. In this example, we assume the DOA error varies form $0^{\circ}$ to $10^{\circ}$, and the number of sampling points is 300 . For the RCB, we set the spherical uncertainty set parameter $\lambda$ equal to $0.35 N$. In Shahbazpanahi's method, the value $\eta=9 \operatorname{tr}\left(R_{s}\right) / N$ is chosen as [24], where $R_{s}$ is the signal covariance matrix. We set the interested robust response region $\theta \in\left[-2^{\circ}, 13^{\circ}\right]$ in Chen's method and our method. Fig. 1 shows the output SINRs versus the 
mismatch angle. One can observe that the SCB is very sensitive to the arrival angle mismatch. The SCB and Shahbazpanahi's method have good performance for small mismatch angle, but they degrade severely for large mismatch angle since the norm of mismatch steering vector being upper bounded. The performance of proposed method and Chen's method are guaranteed for all mismatch angles, but the performance of proposed method is better than the Chen's method. Moreover, when the mismatch angle is small, the SINR of proposed method decreases slightly compared to the SCB because response region is too large. The performance of these beamformers versus the SNR for the fixd mismatch angle $5^{\circ}$ is shown in Fig. 2. In the high SINR region, the output SINRs of SCB and RCB are seriously degraded by the DOA mismatch. However, the proposed method still has good performance compared to Chen's method. The performance of Shahbazpanahi's method also has good performance since the value of $\eta$ is selected properly when the mismatch angle is $5^{\circ}$.

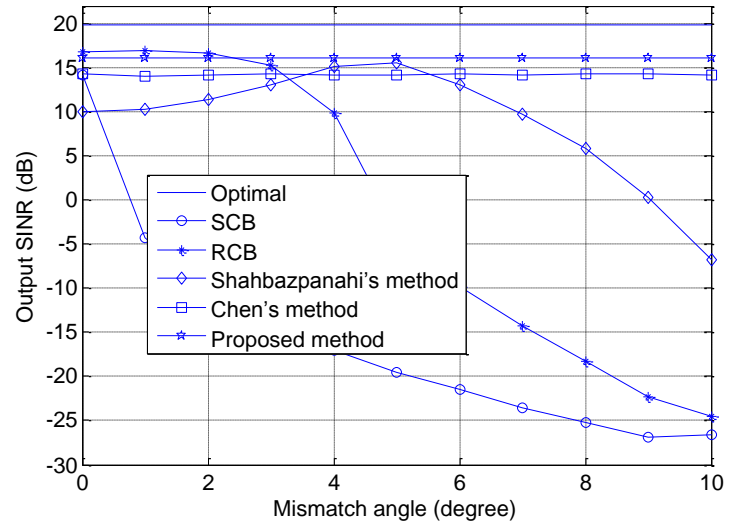

Figure 1. Output SINR versus Mismatch Angle

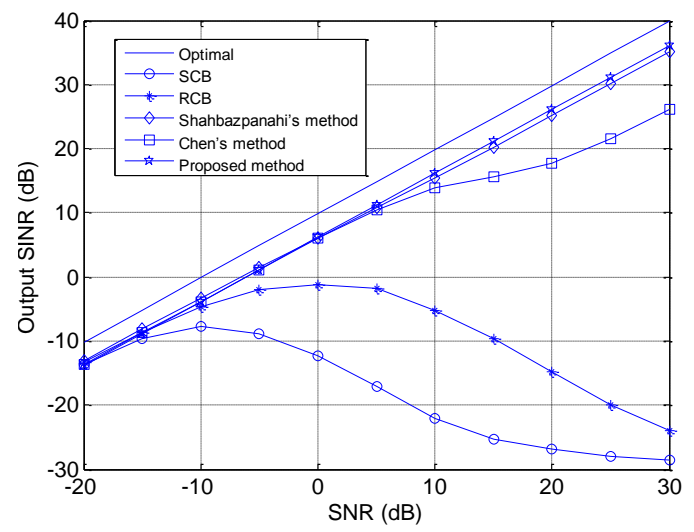

Figure 2. Output SINR versus the SNR for the Fixd Mismatch Angle $5^{\circ}$

Then, we study the sensitivity of output SINR to regularization factor in the proposed method. As before, the interested robust response region is set $\theta \in\left[-2^{\circ}, 13^{\circ}\right]$. Since the parameter $\varepsilon$ changes with the power of input signal, we use a relative regularization factor $\varepsilon_{r}=\varepsilon N / \operatorname{tr}(\hat{\boldsymbol{R}})$. In this experiment, the array is assumed to have random phase errors with standard deviation $\sigma_{\phi}=5^{\circ}$. The relative regularization factor $\varepsilon_{r}$ varies from 0 to 2. Fig. 3 shows the output SINR of proposed method with various numbers of array snapshots versus 
regularization factors. It is clear that even with different number of array snapshots, the performance of proposed method with random phase errors is not very sensitive to the selection of regularization factor. When the array snapshots are larger than the 50, the proposed method has good performance.

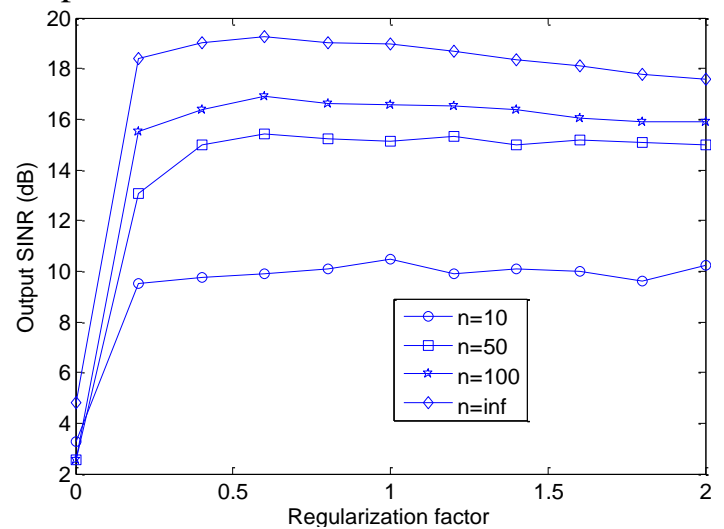

Figure 3. Output SINR of Proposed Method with Different Numbers of Array Snapshots versus Regularization Factors

Moreover, we evaluate the performance of proposed method considering different kinds of array imperfections. Without loss of generality, we only consider the arbitrary array steer vector (ASV) error. In this example, the ASV is modeled as $\overline{\boldsymbol{s}}(\theta)=\boldsymbol{s}(\theta)+\boldsymbol{e}$, where $e$ is a random vector with i.i.d. components $e_{i} \square C N\left(0, \sigma_{e}^{2}\right)$ for all $i$. The $\sigma_{e}^{2}$ varies from 0 to 0.2 . Fig. 4 shows the output SINR of proposed method versus arbitrary ASV error. It can be seen that if the regularization factor is properly selected, the proposed method is robust against these array imperfections. The performance of proposed method with large regularization factor is slightly below the others in the case of small array imperfection. However, to resist significant array imperfections, a large regularization factor is necessary.

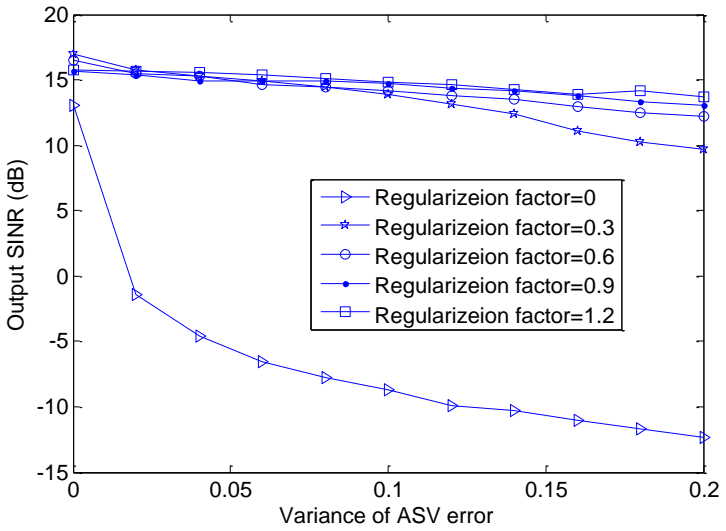
Figure 4. Output SINR of Proposed Method with Various Regularization Factors
versus Arbitrary ASV Error

Finally, the performance of proposed method is compared with the other adaptive beamformers considering various kinds of array imperfections. As before, we consider the arbitrary array steer vector (ASV) error. The simulation parameters are set the same as the first example. Fig. 5 shows the output SINR of these methods versus arbitrary ASV error, in 
which the number of sampling points is 100. It can be seen that the performance of proposed method is guaranteed if a suitable regularization factor is used. The other methods are not very sensitive to the change of array imperfections, but the output SINR of these beamformers is less than the proposed method.

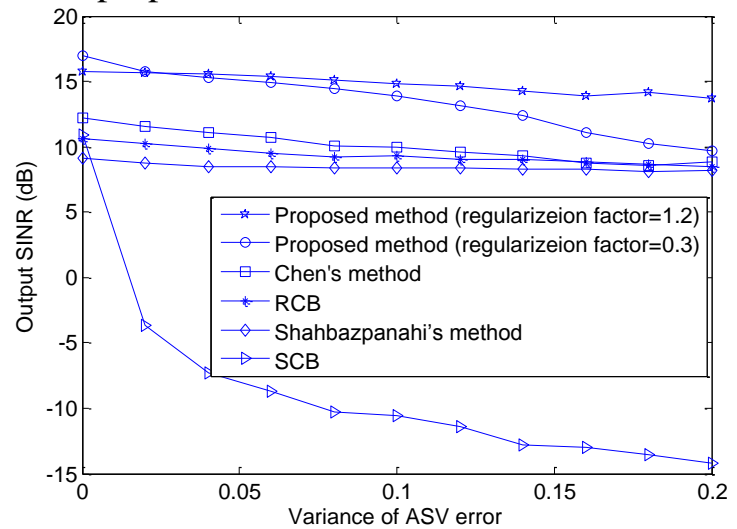

\section{Figure 5. The SINR Outputs of Adaptive Beamformers versus Arbitrary ASV Error}

\section{Conclusions}

In this paper, we formulate the robust beamformer as a semidefinite programming (SDP) problem. With constraints imposed on the magnitude response, the proposed adaptive beamformer can be robust against DOA mismatch. Moreover, the proposed beamformer designed based on worst-case optimization is robust against all kinds of array imperfections. The numerical experiments have been carried out to illustrate the superior performance of the proposed method on output SINR enhancement and robustness against array imperfections. Since the performance of the designed beamformer is not very sensitive to the change of regularization factor, it is very suitable for practical applications.

\section{Acknowledgements}

This work was supported by National Natural Science Foundation of China (Grant No. 61102107, 61374208), the Fundamental Research Funds for the Central Universities (HEUCFX41310).

\section{References}

[1] J. Capon, "High-resolution frequency wavenumber spectrum analysis", IEEE Proc., vol. 57, no. 8, (1969).

[2] J. Li and P. Stoica, "Robust Adaptive Beamforming", Eiley, Hoboken, NJ, (2006).

[3] B. D. Carlson, "Covariance matrix estimation errors and diagonal loading in adaptive arrays", IEEE Trans. Aerospace Electronic System, vol. 23, no. 2, (1988).

[4] O. L. Forst, "An algorithm for linearly constrained adaptive processing", IEEE Proc., vol. 60, no. 8, (1972).

[5] K. Takao, H. Fujita and T. Nishi, "An adaptive arrays under directional constraint", IEEE Trans. Antennas Propagation, vol. 24, no. 5, (1976).

[6] M. H. Er and A. Cantoni, "Derivative constraints for broad-band element space antenna array processors", IEEE Trans. Acoustic Speech Signal Process., vol. 31, no. 6, (1983).

[7] K. M. Buckley and L. J. Griffiths, "An adaptive generalized side lobe canceller with derivative constraints", IEEE Trans Antennas Propagation, vol. 34, no. 3, (1986).

[8] C. Y. Tseng and L. J. Griffiths, "A unified approach to the design of linear constraints in minimum variance adaptive beamformers", IEEE Trans Antennas Propagation, vol. 40, no. 12, (1992). 
[9] F. Quian and B. D. Van Veen, "Quadratically constrained adaptive beamforming for coherent signal and interference", IEEE Trans. Signal Process., vol. 43, no. 8, (1995).

[10] B. D. Van Veen, "Minimum variance beamforming with soft response constraints", IEEE Trans. Signal Process., vol. 39, no. 9, (1991).

[11] K. L. Bell, Y. H. Ephraim and L. V. Trees, "A Bayesian approach to robust adaptive beamforming", IEEE Trans. Signal Process., vol. 48, no. 2, (2000).

[12] D. Feldman and L. Griffiths, "A projection approach for robust adaptive beamforming", IEEE Trans. Signal Process., vol. 42, no. 4, (1994).

[13] L. Chang and C. C. Yeh, "Performance of DMI and eigenspace-based beamformers", IEEE Trans. Antennas Propagation, vol. 40, no. 7, (1992).

[14] S. A. Vorobyov, A. B. Gershman and Z Q. Luo, "Robust adaptive beamforming using worst-case performance optimization: a solution to the signal mismatch problem", IEEE Trans. Signal Processing, vol. 51, no. 2, (2003).

[15] R. G. Lorenz and S. P. Boyd, "Robust minimum variance beamforming”, IEEE Trans. Signal Processing, vol. 53, no. 5, (2005).

[16] J. Li, P. Stoica and Z. Wang, “On Robust Capon Beamforming and Diagonal Loading”, IEEE Trans. Signal Processing, vol. 51, no. 7, (2003).

[17] P. Stoica, Z. Wang and J. Li, "Robust Capon beamforming", IEEE Signal Processing Letter, vol. 10, no. 6, (2003).

[18] C. Y. Chen and P. P. Vaidyanathan, "Quadratically constrained beamforming robust against direction-ofarrival mismatch", IEEE Trans. Signal Process, vol. 55, no. 8, (2007).

[19] S. Boyd and L. Vandenberghe, "Convex Optimization", Cambridge Univ. Press. Cambridge U.K., (2004).

[20] M. Grant and S. Boyd, "CVX: Matlab Software for Disciplined Convex Programming (Web Page and Software)", [Online]. Available: http://stanford.edu/ boyd/cvx(2008).

[21] J. F. Sturm, "Using SeDuMi 1.02, A Matlab toolbox for optimization over symmetric cones", Optim Math Softw., vol. 11, no. 7, (1999).

[22] W. K. Ma, T. N. Davidson, K. M. Wong, Z. Q. Luo and P. C. Ching, "Quasi-ML multiuser detection using semi-definite relaxation with application to synchronous CDMA", IEEE Trans. Signal Processing, vol. 50, no. 4, (2002).

[23] Z. L. Yu, M. H. Er, W. Ser and Z. Gu, "Spectral factorization for integer-interval sampled sequence with applications in array processing", Signal Processing, vol. 88, no. 7, (2008).

[24] S. Shahbazpanahi, A. B. Gershman, Z. Q. Luo and K. M. Wong, "Robust adaptive beamforming for generalrank signal models", IEEE Trans. Signal Process., vol. 51, no. 9, (2003). 
International Journal of Hybrid Information Technology Vol.8, No.2 (2015) 